\title{
Prescription medicine misuse in the Asia-Pacific Region: an evolving issue?
}

\author{
Wui Ling Chan ${ }^{1}$, David Wood ${ }^{2}$, and Paul Dargan ${ }^{2}$ \\ ${ }^{1} \mathrm{Ng}$ Teng Fong General Hospital \\ ${ }^{2}$ Guy's and St Thomas' NHS Foundation Trust
}

July 2, 2020

\begin{abstract}
Abstract Prescription medicine misuse, especially misuse of opioids has become a major public healthcare issue in many developed countries such as the United States of America and Australia where this is associated with significant morbidity (Emergency Department visits due to acute toxicity) and mortality. In this review, we looked at the available data obtained from peer-reviewed articles and population surveys to gain an insight into the current situation in the Asia Pacific region. There is currently limited information available, but data from subpopulation surveys in a number of countries suggests that prescription medicine misuse is likely to be an issue of concern from a public health perspective in the Asia Pacific region. The available data suggest that misuse prevalence rates and the medicines that are commonly misused are similar to countries such as the USA and United Kingdom. Further studies are required to determine the overall prevalence of misuse, the harms associated with this and the sources of drugs being misused so that appropriate interventions can be implemented to tackle issues related to prescription medicine misuse in this region. Keywords: Prescription medicine misuse, drug abuse, opioids, benzodiazepines, GABA analogues
\end{abstract}

\begin{abstract}
Prescription medicine misuse, especially misuse of opioids has become a major public healthcare issue in many developed countries such as the United States of America and Australia where this is associated with significant morbidity (Emergency Department visits due to acute toxicity) and mortality. In this review, we looked at the available data obtained from peer-reviewed articles and population surveys to gain an insight into the current situation in the Asia Pacific region. There is currently limited information available, but data from subpopulation surveys in a number of countries suggests that prescription medicine misuse is likely to be an issue of concern from a public health perspective in the Asia Pacific region. The available data suggest that misuse prevalence rates and the medicines that are commonly misused are similar to countries such as the USA and United Kingdom. Further studies are required to determine the overall prevalence of misuse, the harms associated with this and the sources of drugs being misused so that appropriate interventions can be implemented to tackle issues related to prescription medicine misuse in this region.
\end{abstract}

Keywords:

Prescription medicine misuse, drug abuse, opioids, benzodiazepines, GABA analogues

Introduction

Prescription medicine misuse is the use of medications without a prescription or in a way other than their intended or prescribed indication, or the use of prescription medicines that are sold on the illicit market [1-2]. Using medications at larger doses, more frequent dosing, prolonged duration of use, changing the route 
of administration and/or use together with other medicines (e.g. recreational drugs or novel psychoactive substances) are examples of how medicine can be misused and potentially be harmful or fatal [3].

Prescription medicine, especially that of prescription opioids, has been shown to be a major public healthcare issue in the United State of America (USA) where two thirds of drug overdose deaths were related to opioid use in 2016 [4-6]. An average of 41 people died daily from prescription opioids overdose, totalling nearly 15,000 deaths in the USA in 2018 [7]; and prescription opioids were involved in $32 \%$ of all opioid overdose deaths in 2018 [8].

Prescription medicine misuse is becoming a global concern as data from Australia [9] and Europe [10-12] have shown that these countries are reporting increasing rates of misuse, overdose or death related to misuse of prescription medicine including opioids, benzodiazepines and gabapentinoids.

Unfortunately, the data on prescription medicine misuse in the Asia Pacific region is lacking for the following reasons: 1) there is paucity of national surveys on drug use in general and prescription medicine misuse in particular, 2) most peer-reviewed literature on this topic are from North America, Europe and Australasia and 3) some of the data that are available are published in non-English native languages and are not easily available.

According to a report from the United Nations Office on Drugs and Crime (UNODC) [13], prescription medicine misuse is an increasing phenomenon in South Asia amongst recreational drugs users. In Bangladesh, statistics from the Department of Narcotics Control (DNC) indicate that $12 \%$ of drug users seeking treatment are users of prescription medicines. Medications including psychotropic agents are known to substitute illicit drugs when the supply of illicit drugs is short. In India, among the patients seeking treatment for drug related issues, opiate use accounts for $40 \%$ of the total drug use of which $14.3 \%$ is heroin, $14.2 \%$ is opium and $12.1 \%$ is pharmaceutical opioids.

The factors that have been proposed to contribute to this increase are:

- Prescription medicines can be easily and legally obtained from pharmacies in many countries in the region, often without the need for a prescription.

- There is less stigma associated with the use of prescription medicines compared to the use of recreational drugs. Use of recreational/illicit drugs is still frowned upon in many Asia societies and users can be persecuted for illicit drug use if they are caught by law enforcement in many of the countries in the Asia Pacific region [14].

- Lastly, as seen in North America, Australia and Europe [15], there is an increased prescribing by physicians of opioid analgesics in the management of patients with pain. This increased exposure to opioids has the potential to be associated with an increase in the number of patients developing opioid dependence.

The commonly misused medicines reported in South Asia countries in the UNODC report are similar to that reported in the Western literature. They consist of opioid containing drugs such as codeine containing cough mixtures and opioid analgesics, and benzodiazepines [13].

Data on the worldwide availability of opioids revealed the availability in many countries in Asia Pacific $(0.1-1 \mathrm{mg} /$ capita) is much lesser compared to USA and countries in Europe where the availability of opioids is more than $10 \mathrm{mg}$ per capita in many of the states or countries in these areas where prescription opioids misuse is a growing public and healthcare problem [16].

Literature Review

A systematic search was performed using PubMed and the search strategy included the following keywords: prescription medicine or drugs and misuse or abuse and Asia. The search yield 451 papers, of which we identified the following papers which gave us an insight into the current situation in Asia Pacific, indicating that countries in this region are also facing the similar public healthcare issue with the misuse of medications. The first 2 papers describe the trend of prescription medicine misuses in teenagers/ young adults in China, 
the next 2 are subpopulation surveys on prescription medicine misuse in Thailand and Japan and the last 6 papers describe the misuse of medications especially that of tramadol in the Gulf region.

We shall now look at two papers describing the prevalence of misuse of prescription medicines among students in China. Tam et al [17] reported that from January 2017 to April 2017, 849 undergraduates participated in online survey on non-medical use of prescription medicines. The mean age of the participants was 20 years old and $72.2 \%$ were female. The lifetime and past three months misuse prevalence rate for any prescription medicines were $62.9 \%$ and $33.4 \%$ respectively among 299 students in Beijing and $35.9 \%$ and $21.8 \%$ respectively among 550 students in Macau. The most commonly reported drugs were analgesics (62.9\% Beijing; 35.5\% Macau), followed by sedatives (4.0\% Beijing; 0.9\% Macau), anxiolytics (2.7\% Beijing; $0.6 \%$ Macau), and stimulants (1.0\% Beijing; $0.2 \%$ Macau).

Another study on prescription medicine misuse in the Guangdong Province China [18] was conducted in 2007 to 2009 by Wang Hui et al. This study involved 21,672 middle and high school students whose mean $( \pm \mathrm{SD})$ age was $16( \pm 1.9)$ years. $5.3 \%$ of the female respondents (584 out of 11056 female respondents) and $6.6 \%$ of the male respondents (705 out of 10616 male respondents) reported lifetime misuse of prescription medications. The most common misused medicines were analgesics $(3.9 \%)$, followed by cough medicines with codeine $(2.1 \%)$.

These 2 studies suggest that the prevalence of prescription medicine misuse among adolescents and young adults in China and the types of medications they misuse are similar to the West. There have also been subpopulation surveys on prescription medicine misuse in Japan and Thailand.

The first of these was a random sampling of residents in seven cities/ municipalities in Japan from 2002 to 2004[19]. Face-to-face household surveys were conducted for 887 participants. The lifetime misuse prevalence of prescription medicines was $6.4 \%$ whereas the lifetime use of marijuana and cocaine was $1.5 \%$ and $0.3 \%$ respectively. Misuse prevalence of prescription medicines in the last 12 months was reported to be $1.5 \%$ and use of marijuana was $0.3 \%$. None of the respondents reported use of cocaine in the last 12 months. The study also found that prescription medicine misuse was significantly more common among the middle-aged (35-49 years) group ( $\mathrm{df}=3, \mathrm{P}=0.029)$ and those who were married/cohabitating $(\mathrm{df}=2, \mathrm{P}=0.019)$. Misusers of psychotropic agents were more commonly associated with the following conditions: presence of mood disorder $(\mathrm{OR}=2.82)$, anxiety disorder $(\mathrm{OR}=2.54)$, intermittent explosive disorder $(\mathrm{OR}=3.62)$, and alcohol abuse/ dependence $(\mathrm{OR}=1.99)$ (all $p<0.05)$. The authors commented that although the misuse prevalence rate of prescription medicine was higher than the use of illicit drugs in this study, the overall prevalence for both prescription medicine misuse and illicit drugs use was lower than that reported in countries such as the USA and in Europe. They postulated that this might be the effect of the Japanese government's policy on controlling the use of illicit and other psychotropic drugs.

In a household survey conducted in Thailand in 2007 [20], 26,633 individuals aged between 12 to 65 -yearold participated in a face to face structured interview questionnaire. The lifetime misuse prevalence rate of anxiolytics and hypnotics was $1.58 \%$ whereas the misuse prevalence rate in the last one year was $0.92 \%$ (anxiolytics) and $0.8 \%$ (hypnotics). The findings of this Thai household survey was similar to that from Japan, suggesting that there is an issue with prescription medicine misuse in Thailand - the prevalence rate appears to be lower than that reported in the developed countries but it is important to note that these studies were undertaken a number of years ago.

In India, a retrospective study of 950 patients who were admitted from 2007 to 2014 in a tertiary addiction centre revealed that alprazolam (50.6\%), nitrazepam (23.5\%), and zolpidem $(11.2 \%)$ were the most commonly reported drugs of abuse [21]. High misuse prevalence of benzodiazepines and Z-drugs in this study of patients with drug dependence may suggest that there are significant problems with the misuse of benzodiazepines and Z-drugs in India which is similar to the findings of the UNODC report on prescription medicine misuse in South Asia [13].

Next, we will look at studies from the Iraq, Iran and Yemen to understand the extent of prescription medicine misuse in the Gulf region. The Iraqi Ministry of Health, together with USA government researchers, held an 
Iraqi Community Epidemiology Work Group meeting in May 2012 in Baghdad [22]. This workgroup evaluated information from drug seizure data, records from hospitals, outpatient clinics, and health centres, as well as from surveys of patients, and prisoners. The findings revealed that the most widely misused substance in Iraq was alcohol, followed by prescription drugs such as benzodiazepines, benzhexol and codeine. It was also highlighted that the use of hashish (cannabis resin), tramadol, and amphetamine-type substances (ATS) were increasing in some parts of Iraq.

In a study analysing biological samples obtained during autopsy in Iran to evaluate the trends of tramadolrelated deaths that occurred between 2005 and 2008 in Tehran it was found that tramadol-related deaths were 32.5 more common in 2008 than in 2005, however data were not reported on the circumstances of these deaths and whether they related to deliberate self-poisoning or overdose in the context of tramadol misuse [23].

Another study performed in Iran evaluated the misuse prevalence of tramadol in Iranian adolescents in 2012 [24]. 2000 students participated in this study using a self-administered multiple-choice questionnaire. It was found that the lifetime misuse prevalence of tramadol was $4.8 \%$ ( $7.6 \%$ males; $1.8 \%$ females). Adjusted odds ratios and confidence intervals for lifetime tramadol misusers reporting substance use during the past month were 2.2 (1.1-4.4) for alcohol, 5.0 (1.5-21.9) for cannabis, 8.9 (2.7-29.4) for ecstasy, 0.5 (0.03-7.0) for methamphetamine and 2.3 (0.7-7.4) for opium.

A systematic review of tramadol use and public health consequences by Rostam-Abadi Y et al revealed that the pooled estimates of last 12-month use of tramadol in the Iranian general population were $4.9 \%$ $[95 \%$ confidence interval $(\mathrm{CI})=4.1-5.9]$ among males and $0.8 \%(95 \% \mathrm{CI}=0.2-1.8)$ among females. In the subgroup of Iranian university students, the estimates for last 12 -month use were $4.8 \%(95 \% \mathrm{CI}=1.9-8.9)$ for males and $0.7 \%$ ( $95 \% \mathrm{CI}=0.3-1.1)$ for females. The pooled estimate of tramadol poisoning was $13.1 \%$ $(95 \% \mathrm{CI}=5.7-22.9)$ and the pooled percentage of tramadol-related fatalities was $5.7 \%(95 \% \mathrm{CI}=0.5-15.4)$ among the patients who had drug related overdoses [25].

Another study looking at population size estimation of tramadol misusers in Iran was published in 2019. The surveyed pharmacists estimated that 5.67 people (71560 people) per 1000 population aged 15-49 years were estimated to have misused tramadol [95\% uncertainty limits (UL): 2.61-9.25] whereas the surveyed medicinal herb sellers estimated the abuse rate to be 8.1 people (102230 people) per 1000 population aged $15-49$ years (95\% UL: 5.50-27.35) [26].

These studies suggest that tramadol misuse in Iran is significant and mirrors the trend described in United States of America and China in a recent World Health Organisation (WHO) report [27]. In this report, it is stated that the estimated number of individuals who have used tramadol products non-medically at least once in their lifetime increased from 1,990,000 in 2008 to 2,614,000 in 2011 in the USA; and tramadol was found to be the most commonly used prescription opioid (27.3\%) amongst opioid dependent users in China.

This trend of tramadol misuse was also observed by the pharmacists working in community pharmacies in Aden city, Yemen [28]. 170 pharmacists participated in a questionnaire survey in 2013. 57.7\% of them suspected drug abuse/misuse in their pharmacies and the top three medications for which they suspected misuse were alprazolam, ketoprofen, and tramadol. $83.3 \%$ of them also reported that they observed an increasing pattern of abuse/misuse in their pharmacies.

Prescription Medicine Misuse in Singapore

Singapore is known for its strict legislation on drug use as the country aims to strive for a drug free society through active enforcement against drug activities, preventive education and engagement of the local communities [29]. There is limited literature or population surveys to determine the prevalence of prescription medicine misuse in Singapore.

Newspaper articles describing students misusing medications for attention deficit hyperactivity disorder (ADHD) as a study aid, in the hope of getting better results were published in 2017 [30] and 2013 [31]. These medications can only be prescribed by doctors in Singapore and the article [30] described that students share 
these medications among their peers and it was also possible to obtain these drugs from online platforms and messaging services. Such diversion of drugs is consistent with the global drug trends where more consumers were observed to be using darknet markets to obtain drugs in most countries with over $25 \%$ reporting first time access to Darknet in 2018 in the annual global drug survey report [32].

An article by Winslow et al described the demographics of one hundred and twenty buprenorphine abusers who fulfilled the diagnostic criteria for opiate dependence [33]. The majority of these buprenorphine abusers were male (90\%) with a mean age of 39.2 (SD 8.0) years. $52.5 \%$ were currently employed and $98 \%$ had at least primary education. A family history of drug abuse was reported by $27 \%$. Early onset of Illicit drug abuse occurred at the mean age 16.9 (SD 4.8) years. The finding of this study indicates a possible trend of local drugs misusers moving from abuse of illicit substances to prescription medicines. Understanding such trends in addiction and dependence may help to better tackle the issue of drug misuse locally.

In another paper evaluating prescription medicine misuse among 2273 individuals who were seeking treatment for substances misuse in Singapore [34]. 295 misused prescription medicines only, 811 used illicit drugs, and 263 misused a mixture of both prescription and illicit drugs. 904 were excluded from the study as they reported using alcohol only. It is found that those who were misusing prescription medicines were predominantly male $(243,82.4 \%)$ with a mean age of 41.2 (SD 11.4) years. $63.1 \%$ among this group ( $\mathrm{n}=186)$ reported misusing benzodiazepines as their main drugs and $18.6 \%(n=5)$ used codeine. In the group of illicit drug users, $89.6 \%(\mathrm{n}=727)$ were male with a mean age of 41 (SD 14) years. Heroin was reported to be the main substance used by $63.4 \%(\mathrm{n}=514)$, followed methamphetamines $(18.7 \% ; \mathrm{n}=152)$ and cannabis $(9.1 \% ; \mathrm{n}=74)$. In the group who misused both illicit and prescription medicines, $89 \%(\mathrm{n}=234)$ were male with a mean age of 45.2 (SD 11.2) years. $61.6 \%(\mathrm{n}=162)$ used heroin as their main drug, $14.1 \%(\mathrm{n}=37)$ misused benzodiazepines, and $11.8 \%(\mathrm{n}=31)$ used amphetamines. It was found that educational level and occupational status were associated with different types of misuse [34]. Those with primary and secondary education qualifications had higher odds (2.1 and 2.9 times, respectively) of misusing both prescriptions and illicit drugs than those with tertiary qualification and those working in managerial or professional roles had higher odds (4.4 and 7.4 times, respectively) of misusing prescription medicines only than those who were unemployed [34].

Due to the paucity of data on the prevalence of prescription medicine misuse in Singapore, we conducted two online internet surveys in 2015 [35] and 2016. The aim of the studies was to determine i) whether survey respondents had heard of different prescription medicines, ii) the prevalence of prescription medicine misuse in Singapore, and iii) identify the demographic characteristics of those reporting misuse. The demographics of the respondents in 2015 and 2016 are illustrated in table 1. The level of awareness and lifetime misuse prevalence for each of the group of medicines surveyed, together with the mean age of the misusers can be found in table 2 .

To compliment the population survey, we conducted an online survey to determine the level of awareness of prescription medicine misuse of doctors in Singapore in 2016[36]. 102 doctors working in the Emergency Department participated in this online survey, of which $55.9 \%$ were male, $54.5 \%$ were consultants and the median (IQR) age was 38 (25-55) years old.

The results of the study showed majority of the respondents $(76.5 \%)$ were concerned about misuse of prescription drugs by their patients. $86.3 \%$ were aware that they might be prescribing to patients who were misusing medicines. $80.8 \%$ felt that they needed help to deal with patients who were misusing prescription medicines and $65.7 \%$ indicated that more training in the management of patients who were prescription medicines misusers was required, of which $15.1 \%$ preferred face-to-face training, $37.9 \%$ preferred online training and $47.0 \%$ were keen for both face-to-face and online training. The top three prescription medicines that the respondents suspected that their patients might be misusing were: codeine containing cough mixtures $(92.2 \%)$, opioids analgesics $(83.8 \%)$ and benzodiazepines $(64.7 \%)$

The findings of this survey are consistent with the self-reported misuse prevalence rates of the earlier online surveys in Singapore and the UNODC report [13] where the most commonly misused medications in South 
Asia were codeine containing cough mixtures, opioid analgesia and benzodiazepines. In additional, the data from the online survey conducted in 2015 and 2016 suggests that prescription medicine misuse may be an issue in Singapore and would require further studies such as general population survey to look at the extent of misuse in the general public and to further evaluate the sources of drug diversions.

Despite the limited data at a population level in many countries in the Asia Pacific region, we can suggest with current available data, particularly from Singapore, that prescription medicine misuse is likely to be a problem in the region.

More research is required to understand the scale of the problem, demographics and reasons for misuse and the sources of availability. This additional information will help determine if changes to prescribing practices with regular audit and monitoring of physicians' habits, implementation of preventive educational activities to raise awareness of the dangers of prescription medicine misuses, provision of services for drug treatment and rehabilitation and revision of legislation to tighten control are required. These changes will eventually help reduce or prevent prescription medicine misuse from becoming a public healthcare burden in this region.

References

1. National Institute on Drug Abuse. Misuse of prescription drugs. Available at https://www.drugabuse.gov/publications/misuse-prescription-drugs/overview. Last accessed 28 June 2020

2. World Health Organization (WHO). Lexicon of Alcohol and Drug Terms Published by the World Health Organization. 2006. [accessed 2006]. Available at: http://www.who.int/substance_abuse/terminology/who_lexicon/en/. Last accessed 28 June 2020

3. Statistics in Drug Misuse, England, 2019: Death related to poisoning by drug abuse. Available at https://digital.nhs.uk/data-and-information/publications/statistical/statistics-on-drug-misuse/2019/part2-deaths-related-to-poisoning-by-drug-misuse. Last accessed 28 June 2020

4. CDC: Overdose Deaths Involving Opioids, Cocaine, and Psychostimulants - United States, 2015-2016. Available at https://www.cdc.gov/mmwr/volumes/67/wr/mm6712a1.htm Last accessed 28 June 2020

5. International Statistics. Available at https://www.drugfreeworld.org/drugfacts/prescription/abuseinternational-statistics.html. Last accessed 28 June 2020

6. Seth $\mathrm{P}$ et al. Quantifying the Epidemic of Prescription Opioid Overdose Deaths. American Journal of Public Health. March 2018; 108(4)e1-e3

7. Wilson N, Kariisa M, Seth P, et al. Drug and Opioid-Involved Overdose Deaths-United States, 20172018. MMWR Morb Mortal Wkly Rep 2020;69:290-297

8. Centres for Disease Control and Prevention: Opioid overdose. Available at https://www.cdc.gov/drugoverdose/data/prescribing/overdose-death-maps.html. Last accessed 28 June 2020

9. National Drug Strategy Household Survey 2016: Misuse of Pharmaceuticals. Available at https://www.aihw.gov.au/getmedia/15db8c15-7062-4cde-bfa4-3c2079f30af3/21028a.pdf.aspx?inline=true.

Last accessed 28 June 2020

10. Kapil V, Green JL, Le Lait C, Wood DM, Dargan PI. Misuse of benzodiazepines and Z-drugs in the UK. Br J Psychiatry 2014; 205:407-8

11. Kapil V, Green JL, Le Lait MC, Wood DM, Dargan PI. Misuse of the gamma-aminobutyric acid analogues baclofen, gabapentin and pregabalin in the UK. Br J Clin Pharmacol 2014; 78:190-1

12. Weisberg DF, Becker WC, Fiellin DA, Stannard C. Prescription opioid misuse in the United States and the United Kingdom: cautionary lessons. The International Journal on Drug Policy. 2014;25(6):1124-1130. doi:10.1016/j.drugpo.2014.07.009 
13. UNODC: Misuse of prescription drugs: A South Asia perspective. Available at https://www.unodc.org/documents/southasia/reports/Misuse_of_Prescription_Drugs_-_A_South_Asia_Perspective_UNODC_2011.pdf. Last accessed 28 June 2020

14. Drug Abuse: The 20 Countries with the Harshest Drug Laws in the World. Available at https://drugabuse.com/the-20-countries-with-the-harshest-drug-laws-in-the-world/. Last accessed 28 June 2020

15. American Academy of Family Physicians (AAFP): Pain management and opioid misuse. Available at https://www.aafp.org/patient-care/public-health/pain-opioids.html. Last accessed 28 June 2020

16. Worldwide availability of opioids per capita. Available at https://www.uwhealth.org/news/clearyopiods/47671. Last accessed 28 June 2020

17. Tam CC, Benotsch EG, Wang X, Lin D, Du H, Chi P. Non-medical use of prescription drugs and cultural orientation among college students in China. Drug and Alcohol Dependence. 2018;192:271-276. doi:10.1016/j.drugalcdep.2018.08.012

18. Wang H, Deng J, Zhou X, et al. The nonmedical use of prescription medicines among high school students: a cross-sectional study in Southern China. Drug and Alcohol Dependence. 2014;141:9-15. doi:10.1016/j.drugalcdep.2014.04.004

19. Tominaga M, Kawakami N, Ono Y, et al. Prevalence and correlates of illicit and non-medical use of psychotropic drugs in Japan: findings from the World Mental Health Japan Survey 2002-2004. Social Psychiatry and Psychiatric Epidemiology. 2009;44(9):777-783. doi:10.1007/s00127-009-0499-1

20. Assanangkornchai S, Sam-Angsri N, Rerngpongpan S, Edwards JG. Anxiolytic and hypnotic drug misuse in Thailand: findings from a national household survey. Drug and Alcohol Review. 2010;29(1):101-111. doi:10.1111/j.1465-3362.2009.00092.x

21. Shukla L, Bokka S, Shukla T, et al. Benzodiazepine and "Z-Drug" Dependence: Data From a Tertiary Care Center. The Primary Care Companion for CNS disorders. 2017;19(1):10.4088/PCC.16br02025. Published 2017 Feb 16. doi:10.4088/PCC.16br02025

22. Al-Hemiary NJ, Al-Diwan JK, Hasson AL, Rawson RA. Drug and alcohol use in Iraq: findings of the inaugural Iraqi Community Epidemiological Workgroup. Substance Use and Misuse. 2014;49(13):1759-1763. doi:10.3109/10826084.2014.913633

23. Iravani FS, Akhgari M, Jokar F, Bahmanabadi L. Current trends in tramadol-related fatalities, Tehran, Iran 2005-2008. Substance Use and Misuse. 2010;45(13):2162-2171. doi:10.3109/10826081003692098

24. Nazarzadeh M, Bidel Z, Carson KV. The association between tramadol hydrochloride misuse and other substances use in an adolescent population: Phase I of a prospective survey. Addictive Behaviors. 2014;39(1):333-337. doi:10.1016/j.addbeh.2013.09.013

25. Rostam-Abadi Y, Gholami J, Amin-Esmaeili M, et al. Tramadol use and public health consequences in Iran: a systematic review and meta-analysis [published online ahead of print, 2020 Mar 20]. Addiction. 2020;10.1111/add.15059. doi:10.1111/add.15059

26. Nasiri N, Abedi L, Hajebi A, et al. Population Size Estimation of Tramadol Misusers in Urban Population in Iran: Synthesis of Methods and Results. Addiction and Health. 2019;11(3):173-182. doi:10.22122/ahj.v11i3.240

27. WHO Critical Review Report: Tramadol: Non-medical use, abuse and dependence. Available at https://www.who.int/medicines/access/controlled-substances/Tramadol.pdf?ua=1. Last accessed 28 June 2020

28. Abood EA, Wazaify M. Abuse and Misuse of Prescription and Nonprescription Drugs from Community Pharmacies in Aden City-Yemen. Substance Use and Misuse. 2016;51(7):942-947. 
doi:10.3109/10826084.2016.1155619

29. Central Narcotics Bureau. Available at https://www.cnb.gov.sg/about-us\#vision. Last accessed 28 June 2020

30. The Straits Times. Students popping 'smart pills' to lift grades. Available at https://www.straitstimes.com/singapore/education/students-popping-smart-pills-to-lift-grades. Last accessed 28 June 2020

31. The Straits Times. Students fake ADHD symptoms to get 'brain booster' drug. Available at https://www.straitstimes.com/singapore/students-fake-adhd-symptoms-to-get-brain-booster-drug. Last accessed 28 June 2020

32. Global Drug Survey. Executive Summary 2019. Available at https://www.globaldrugsurvey.com/wpcontent/themes/globaldrugsurvey/results/GDS2019-Exec-Summary.pdf. Last accessed 28 June 2020

33. Winslow M, Ng WL, Mythily S, Song G, Yiong HC. Socio-demographic profile and help-seeking behaviour of buprenorphine abusers in Singapore. Annals of the Academy of Medicine Singapore. 2006;35(7):451-456

34. Pv A, Abdin E, Jun Wen T, Subramaniam M, Cheok C, Song G. Correlates of Non-Medical Prescription Drug Misuse Among a Treatment-Seeking Population: A Comparison with Illicit Drug Users. International Journal of Environmental Research and Public Health. 2018;15(9):1978. Published 2018 Sep 11. doi:10.3390/ijerph15091978

35. Chan WL, Dargan PI, Haynes CM, et al. Misuse of prescription medicines is as prevalent as the use of recreational drugs and novel psychoactive substances in Singapore: an unrecognised public health issue? [published online ahead of print, 2020 Mar 17]. Singapore Medical Journal. 2020;10.11622/smedj.2020024. doi:10.11622/smedj.2020024

36. WL Chan, DM Wood, PI Dargan. Doctors' Awareness and Perception of Prescription Medicine Misuse in Singapore. 38th International Congress of the European Association of Poisons Centres and Clinical Toxicologists (EAPCCT) 22-25 May 2018, Bucharest, Romania, Clinical Toxicology, 56:6, 453-608, DOI: $10.1080 / 15563650.2018 .1457818$

Bottom of Form

\section{Hosted file}

Tables.docx available at https://authorea.com/users/339096/articles/465375-prescriptionmedicine-misuse-in-the-asia-pacific-region-an-evolving-issue 